Portland State University

PDXScholar

$12-1-2005$

\title{
Investigation of the Probe-Sample Interaction in the Ultrasonic/Shear-Force Microscope: The Phononic Friction Mechanism
}

Xiquan Cui

Portland State University

Andres H. La Rosa

Portland State University, andres@pdx.edu

Follow this and additional works at: https://pdxscholar.library.pdx.edu/phy_fac

Part of the Physics Commons

Let us know how access to this document benefits you.

Citation Details

Cui, X., \& La Rosa, A. (2005). Investigation of the probe-sample interaction in the ultrasonic/shear-force microscope: The phononic friction mechanism. Applied Physics Letters, 87(23), 231907.

This Article is brought to you for free and open access. It has been accepted for inclusion in Physics Faculty Publications and Presentations by an authorized administrator of PDXScholar. Please contact us if we can make this document more accessible: pdxscholar@pdx.edu. 


\title{
Investigation of the probe-sample interaction in the ultrasonic/shear-force microscope: The phononic friction mechanism
}

\author{
Xiquan $\mathrm{Cui}^{\mathrm{a})}$ and Andres La Rosa ${ }^{\mathrm{b})}$ \\ Department of Physics, Portland State University, Portland, Oregon 97201
}

(Received 20 June 2005; accepted 25 October 2005; published online 29 November 2005)

\begin{abstract}
The dissipative and conservative interactions between a sharp probe and a flat Si sample in the ultrasonic/shear-force microscope are investigated. It is shown that, when working in the ambient condition, there are two distinct probe-sample interaction regions: the pure dissipative interaction region in the relatively far probe-sample distance, and the highly correlated dissipative and conservative interaction region in the close probe-sample distance. The ultrasonic data suggest that the phonon generation is a dissipative channel for the probe-sample interaction in the shear force microscope. A shaking potential model is proposed to explain the phononic friction mechanism. (C) 2005 American Institute of Physics. [DOI: 10.1063/1.2138793]
\end{abstract}

The shear force is widely used for controlling the probesample distance in the near field scanning optical microscope (NSOM). ${ }^{1}$ In 1995, the quartz tuning fork (TF), was introduced in the shear force microscope. ${ }^{2}$ Because of its high $Q$, high stiffness, simple implementation of the detection system, and no hazard to photon sensitive material, the TF is widely used as a force sensor in a variety of dynamic force microscopes. ${ }^{3}$ But the origin of the shear force interaction is still not well understood. ${ }^{4-8}$

On the other hand, in recent years, due to the development of new interfacial friction tools, significant experimental and theoretical progresses have been made in the area of nanotribology. The new phononic friction model shows that when the wear is negligible in the friction process the phonon generation accounts for the dissipation. ${ }^{9,10}$ But, to the best of our knowledge, no experiment has been implemented to prove the mechanism of phononic friction directly. Here, we incorporate an ultrasonic sensor in the tuning-fork based shear-force microscope to obtain extra information about the probe-sample interaction. The data presented in this manuscript suggest that the phonon generation is a dissipative channel for the wear-free probe-sample shear force interaction.

A more detailed description of the ultrasonic/shear force microscope experimental setup has been provided elsewhere. ${ }^{11}$ Very succinctly (see also the inset in Fig. 1), the sample is a piece of polished $\mathrm{Si}$ wafer, and the probe is a tapered optical fiber with a tip radius of around $30 \mathrm{~nm}$. The resonant frequency of the TF shifts to a lower frequency $31283 \mathrm{~Hz}$ when the probe is attached to it, and its $Q$ decreases to $\sim 10^{3}$. To evaluate the probe-sample interaction at different separation distances, the frequency spectrum of the TF admittance is measured by the lock-in amplifier No. 1; additional information is provided by the ultrasonic transducer placed in intimate contact with the sample, whose output signal is processed by the lock-in amplifier No. 2 .

The motion of the TF can be described by the Newton's equation $\quad M \ddot{x}=F_{\text {drive }}+F_{\text {damp }}+F_{\text {restore }}=F_{\text {drive }}-M \gamma_{0} \dot{x}-k_{0} x$, where $x$ is the displacement of the TF vibration, $F_{\text {damp }}$ is the damping force, $F_{\text {restore }}$ is the restoring force due to the TFs

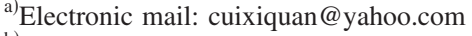

${ }^{b}$ Electronic mail: andres@pdx.edu
}

elastic deformation, $M$ is the effective mass, $\gamma_{0}$ is the damping rate of the free TF in the air, and $k_{0}$ is the effective spring constant of the TF.

The dissipative and conservative probe-sample interaction, $F_{\text {dissipate }}$ and $F_{\text {conserve, }}$, will contribute to the TF motion as follows: $M \ddot{x}=F_{\text {drive }}+F_{\text {damp }}+F_{\text {dissipate }}+F_{\text {restore }}+F_{\text {conserve, }}$ or

$$
\begin{aligned}
M \ddot{x}= & F_{\text {drive }}-M\left(\gamma_{0}+\gamma^{\prime}\right) \dot{\mathrm{x}}-\left(k_{0}+k^{\prime}\right) x \\
& =F_{\text {drive }}-M \gamma \dot{x}-k x,
\end{aligned}
$$

where $\gamma^{\prime}$ is the effective damping rate due to the dissipative interaction, $k^{\prime}$ is the force gradient due to the conservative interaction, $\gamma$ is the total damping rate, and $k$ is the total restoring force gradient.

The electrical response of the TF can be linked to the mechanical response by the piezoelectromechanical coupling constant $\alpha,{ }^{12}$ and $L \ddot{Q}+R Q+(1+C) Q=V_{d}$, where $Q=2 \alpha x, L$ $=M / 2 \alpha^{2}, R=M \gamma / 2 \alpha^{2}, 1 / C=k / 2 \alpha^{2}$, and $V_{d}=F_{\text {drive }} / \alpha$. Due to the parallel capacitance $C_{p}$ of the TF, the electrical admittance of the TF is
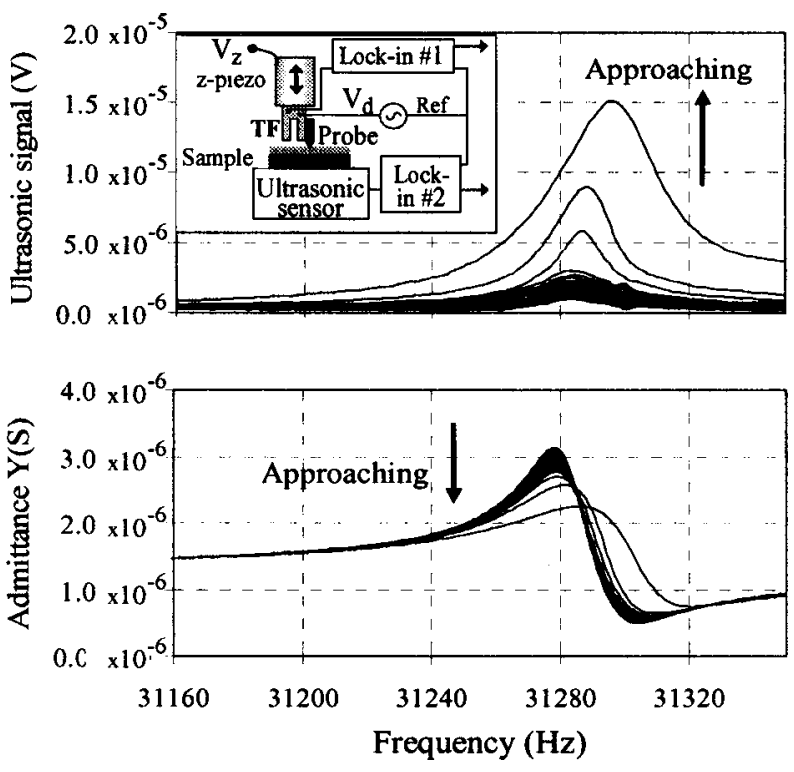

FIG. 1. Variation of the ultrasonic spectra (top) and the TF admittance spectra (bottom), under $60 \mathrm{mV}$ TF driving voltage. The inset shows a schematic of the experimental setup. 


$$
Y(\omega)=\frac{1}{R+i \omega L+\frac{1}{i \omega C}}+i \omega C_{p}
$$

By fitting the admittance of the TF (taken when the tip was away from the samples surface), a set of $L, R, C, C_{p}$ can be determined. For the TF used here $(l=4 \mathrm{~mm}, t=0.6 \mathrm{~mm}, w$ $=0.33 \mathrm{~mm}), k_{\text {bareTF}}=(E / 4) w(t / L)^{3}=22 \times 10^{3} \mathrm{~N} / \mathrm{m}$. The fitting gives $C=1.135 \times 10^{-14} \mathrm{~F}$, from which, according to the equation $1 / C=k / 2 \alpha^{2}$, the piezoelectromechanical coupling constant $\alpha$ of the TF in our experiment is $\alpha=11$ $\times 10^{-6} \mathrm{C} / \mathrm{m}$.

In the steady state of the TF vibration, the time average power consumed by the dissipative probe-sample interaction can be calculated using the mechanical model and the equivalent circuit model separately, which is

$$
P_{\text {dissipate }}=-\frac{2\left(F_{\text {drive }}^{\mathrm{RMS}}\right)^{2} \gamma^{\prime}}{M\left[\left(\frac{\omega_{0}^{2}}{\omega}-\omega\right)^{2}+\gamma^{2}\right]}=-\frac{\left(V_{d}^{\mathrm{RMS}}\right)^{2}\left(R-\mathrm{R}_{0}\right)}{L^{2}\left(\frac{\omega_{0}^{2}}{\omega}-\omega\right)^{2}+R^{2}},
$$

where $R_{0}$ is the equivalent resistance of the TF when it is far away from the probe-sample interaction region. The dissipative power has a peak at the resonant frequency

$$
\omega_{0}^{2}=k / M=1 /(L C) .
$$

Figure 1 shows the TF admittance spectrum under the 60 $\mathrm{mV}$ driving voltage in the approaching process. The closer the TF is to the sample, the stronger the probe-sample interaction becomes. The dissipative interaction, responsible for the damping of the admittance spectrum, increases monotonously. The conservative interaction, responsible for the resonance frequency shift in the admittance spectrum, does not change at the beginning, but increases dramatically at short probe-sample distances. When the probe-sample interaction is so strong that the motion of TF can not be described by a simple harmonic motion, the TF admittance curve will be distorted. Before this critical point, all of the TF admittance curves can be fitted perfectly by Eq. (2). So it is reasonable to take that point as the position of the sample's surface $(z=0 \mathrm{~nm})$.

The ultrasonic spectra are recorded simultaneously with the TF admittance spectra (shown also in Fig. 1). During the approach the ultrasonic spectrum does not change significantly when the probe-sample distance is far, but its peak increases dramatically when the probe-sample distance is very close, thus displaying a correlation with the increasing TFs energy dissipation.

In Fig. 2, the ultrasound and the TF dissipative power spectra are compared when the probe-sample distance is $z$ $\approx 0.5 \mathrm{~nm}$. By choosing a proper ultrasonic signal detection efficiency factor to scale the ultrasonic signal (it is due to the technical difficulties of measuring the exact ultrasound power), the ultrasonic signal can be fitted with the TF dissipative power [Eq. (3)] within the experimental error.

From the energy conservation point of view, the coincidence suggests that the power of the ultrasound generation should come from the TF dissipation. It is consistent with the current phononic friction theory-when the wear is negligible in a friction process the phonon generation accounts for the dissipation. The TF dissipative power is released into the sample by the frictional probe-sample interaction in the form

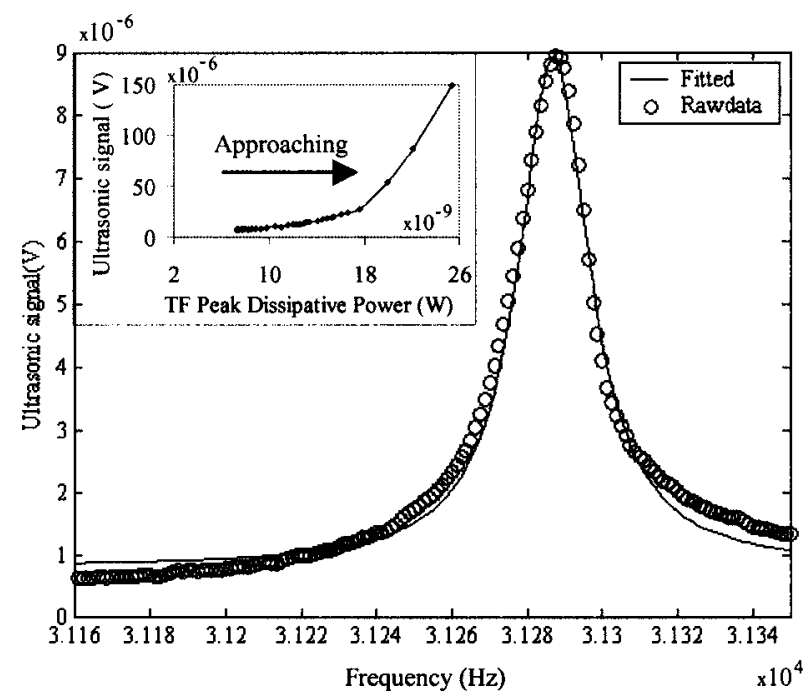

FIG. 2. The ultrasonic spectrum (acquired at $z \approx 0.5 \mathrm{~nm}$ ) fits (apart from a proper scaling factor) the dissipative power of the TF [Eq. (3)]. The inset shows the increase in ultrasonic signal as the TF dissipative power increases.

of phonons, which will either survive and propagate away or dissipate in the form of heat. Maybe there are some other dissipation channels involved, like the excitation of electronhole pairs. ${ }^{9}$ or interactions mediated by the contamination layer. ${ }^{11}$ But the ultrasonic data support that the phonon generation is a dissipation channel in the shear force interaction.

The inset in Fig. 2 shows the increase of the ultrasonic signal peak with the TF dissipative power at the resonant frequency during the probe approaching process under 60 $\mathrm{mV}$ driving voltage. Notice the two distinct regions for the ultrasound generation. The critical point is around $z \approx 1 \mathrm{~nm}$. When the probe-sample distance is greater than $1 \mathrm{~nm}$, the ultrasound generation is proportional to the TF dissipative power with a smaller slope. When the probe-sample distance is less than $1 \mathrm{~nm}$, the slope becomes markedly increases. These two distinct ultrasound generation regions suggest that there are two different types of probe-sample interactions. The observed experimental linear relationship between the ultrasonic signal and the TF dissipative power confirms further their direct correlation.

Figure 3 shows the effective damping rate, the force gradient, and the ultrasonic signal change with respect to the probe-sample distance $z$ under $60 \mathrm{mV}$ driving voltage. The changes in the probe-sample distance are obtained from the $z$ piezodriving voltage measurements. When the probe is at several hundred nanometers away from the sample, the damping rate linearly increases as the probe-sample distance decreases. But neither the force gradient nor the ultrasonic signal change significantly. It means that the probe-sample interaction in this region is mainly dissipative; the contamination layer (water or hydrocarbon compound layer) could be accounted for the viscous dissipation.

When the probe-sample distance is less than $1 \mathrm{~nm}$, the damping rate of the $\mathrm{TF}$, the force gradient and the ultrasonic signal increase dramatically. What kind of forces account for the close probe-sample interaction? It could be atomic force. According to Ref. 4, the Coulomb force and the capillary force are also plausible candidates. The contamination layer could be the reason too, if it could change its viscosity and shear modulus in depth as proposed in Refs. 8 and 11. But the fingerprint of the shear probe-sample interaction in this 


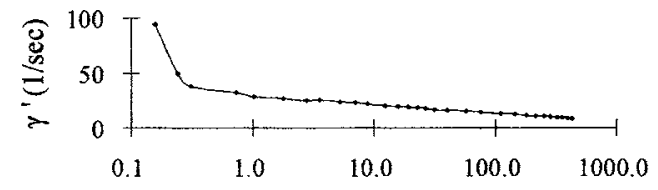

(a)

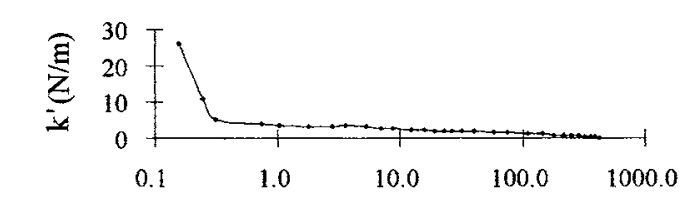

(b)

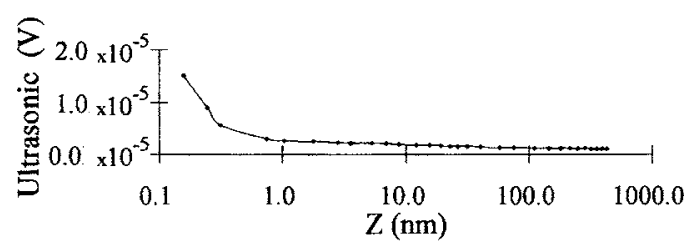

(c)

FIG. 3. The effective damping rate (a), force gradient (b), and ultrasonic signal (c) change with respect to the probe-sample distance $z$ under $60 \mathrm{mV}$ driving voltage.

region is that the dissipative and conservative probe-sample interactions are correlated to each other. The stronger the conservative probe-sample interaction is, the stronger the dissipative probe-sample interaction will be.

Based on the latter observation, we propose a shaking potential model for the phononic friction mechanism of the shear force probe-sample interaction. When the probesample interaction is weak, the probe-sample interaction potential $U(x)$ is approximately quadratic, where $x$ is the lateral probe-sample interaction distance. The second derivative of the interaction potential $k=\partial^{2} U / \partial x^{2}$ causes the resonant frequency change of the $\mathrm{TF}$, and $M \ddot{x}+M \gamma_{0} \dot{x}+k_{0} x=F_{\text {drive }}$ $+F_{\text {probe-sample }}=F_{\text {drive }}-k x$. In previous studies the probesample interaction is considered stationary and conservative. But in fact the probe-sample interaction is a dynamic process, because the sample is plucked by the probe-sample interaction (as confirmed by our ultrasound recording). If the displacement of the sample is $u$, the motion of TF can be expressed as $M \ddot{x}+M \gamma_{0} \dot{x}+k_{0} x=F_{\text {drive }}+F_{\text {probe-sample }}=F_{\text {drive }}$ $-k(x-u)$. Since the displacement of the sample is induced by the motion of TF, it is reasonable to assume $u=\widetilde{A} x=|\widetilde{A}| e^{i \phi} x$, where $\tilde{A}$ is a complex factor describing the coupling between the probe and sample motion. Thus the last equation can be rewritten as

$$
M \ddot{x}+M \gamma_{0} \dot{x}+k_{0} x=F_{\text {drive }}-k(1-|\widetilde{A}| \cos \phi) x+k \frac{|\widetilde{A}| \sin \phi}{\omega} \dot{x} .
$$

According to Eq. (1), $\gamma^{\prime}=(-|\widetilde{A}| \sin \phi / M \omega) k$. This shows that the dissipative probe-sample interaction increases with respect to the conservative probe-sample interaction, which is supported by our experimental data. So in the close probesample interaction region, because of the phase lag of the probe-sample interaction, the conservative interaction is always accompanied by the dissipative interaction, and the dissipative power of $\mathrm{TF}$ is released in the form of phonons.

All in all, the ultrasonic/shear-force microscope has many advantages in nanotribology. By using the mechanical resonator model, a wealth of information about the probesample interaction can be retrieved from the TF and the ultrasound data. When working in the ambient environment, which is very common in NSOM applications, two distinct probe-sample interaction regions are identified. In the closer to the surface region, the shaking potential model, introduced here, provides a new way to explain the correlation between the dissipative and conservative probe-sample interaction, and the phononic dissipation of the shear force interaction.

${ }^{1}$ E. Betzig, P. L. Finn, and J. S. Weiner, Appl. Phys. Lett. 60, 2484 (1992). ${ }^{2}$ K. Karrai and R. D. Grober, Appl. Phys. Lett. 66, 1842 (1995).

${ }^{3}$ F. J. Giessibl, M. Herz, and J. Mannhart, Proc. Natl. Acad. Sci. U.S.A. 99, 12006 (2002).

${ }^{4}$ M. P. Bernal, F. M. Weible, P. Y. Boillat, and P. Lambelet, Proc. IEEE 88, 1460 (2000).

${ }^{5}$ M. J. Gregor, P. G. Blome, J. Schofer, and R. G. Ulbrich, Appl. Phys. Lett. 68, 307 (1996).

${ }^{6}$ K. Hsu and L. A. Gheber, Rev. Sci. Instrum. 70, 3609 (1999).

${ }^{7}$ P. K. Wei and W. S. Fann, J. Appl. Phys. 87, 2561 (2000).

${ }^{8}$ K. Karrai and I. Tiemann, Phys. Rev. B 62, 13174 (2000).

${ }^{9}$ B. N. J. Persson and R. Ryberg, Phys. Rev. B 32, 3586 (1985).

${ }^{10}$ J. Krim, Sci. Am. 275, 74 (1996).

${ }^{11}$ A. La Rosa, R. Nordstrom, X. Cui, J. McCollum and N. Li, Rev. Sci. Instrum. 76, 093707 (2005)

${ }^{12}$ J. Rychen, T. Ihn, P. Studerus, A. Herrmann, and K. Ensslin, Rev. Sci. Instrum. 71, 1695 (2000). 
Applied Physics Letters is copyrighted by the American Institute of Physics (AIP). Redistribution of journal material is subject to the AIP online journal license and/or AIP copyright. For more information, see http://ojps.aip.org/aplo/aplcr.jsp 